\title{
Bras court du chromosome 11 : empreinte parentale différentielle, tumorigenèse et pertes d'allèles
}

Il existe au moins trois gènes de prédisposition au néphroblastome (tumeur de Wilms) dont deux, situés sur le bras court du chromosome 11, sont également impliqués dans des syndromes malformatifs. Au minimum deux événements sont nécessaires à l'apparition d'une tumeur. L'analyse de tumeurs héréditaires, dans lesquelles la première anomalie est d'origine germinale, montre que la deuxième anomalie peut se produire sur un locus différent. Cette deuxième anomalie est habituellement une perte d'allèles maternels. Cette observation confirme la non-équivalence des génomes parentaux et indique que des phénomènes d'empreintes parentales différentielles interviennent dans les mécanismes génétiques de ces tumeurs.

\section{Claudine Junien Isabelle Henry}

\section{ADRESSES}

Cl. Junien : professeur de génétique à ParisOuest, université R. Descartes. I. Henry : chargée de recherche à l'Inserm. Inserm U. 73, château de Longchamp, Bois de Boulogne,

a notion d'anti-oncogène, dont l'archétype est le gène du rétinoblastome $(R B 1)$, repose essentiellement sur la mise en évidence, au niveau moléculaire, de l'altération ou de la perte des deux allèles de ce gène dans une tumeur, introduisant ainsi la notion de caractère récessif pour ce type de mutation [1]. Le clonage du gène $R B 1$, localisé en $13 q 14[2,3]$, a en effet permis de vérifier l'hypothèse de Knudson (1971) [4] qui postulait, au minimum, deux événements pour qu'un rétinoblastome apparaisse. Selon cette «théorie des deux événements », la première mutation devait être germinale pour les cas héréditaires ou bien somatique pour les cas sporadiques, la seconde mutation étant nécessairement somatique dans les deux cas. En 1983, grâce à des marqueurs polymorphes de l'ADN situés de part et d'autre de la région $13 \mathrm{ql} 14$, où se trouve le gène $R B 1$, Cavenee et al. [5] montraient que la deuxième mutation pouvait être due à des remaniements chromosomiques mitotiques (figure 1) aboutissant à une «perte de l'hétérozygotie » appelée encore «perte d'allèles» pour la région portant le gène $R B 1$. Ces expériences, ainsi que la mise en évidence de remaniements moléculaires dans des tumeurs, confirmaient donc l'hypothèse de Knudson, mais également la proposition de Comings selon laquelle les deux événements devaient toucher les deux allèles d'un même gène [6]:

Tout portait à croire que le gène responsable de la prédisposition au néphroblastome, $W T 1$, situé en 1 lpl3, se comportait exactement de la même façon que le gène $R B 1$ : des pertes d'allèles faisant intervenir les mêmes mécanismes chromosomi- 
ques étaient mises en évidence pour le chromosome 11 dans le néphroblastome [7].

\section{Perte préférentielle des allèles maternels}

Selon la théorie de Knudson, on pouvait donc s'attendre à ce que le chromosome restant dans une tumeur soit le chromosome porteur de la première mutation, c'est-à-dire, pour les formes familiales, le chromosome transmis par le parent atteint. Ceci a été vérifié pour des formes familiales de rétinoblastome dans lesquelles le chromosome restant dans la tumeur provenait indiscutablement du parent lui-même atteint [8]. Il semblait logique que cela s'applique également aux formes sporadiques non héréditaires avec, selon le hasard des mutations, tantôt l'allèle paternel, tantôt l'allèle maternel.
Dans le cas du néphroblastome, des études [9-12] portant sur l'identification de l'origine parentale de l'allèle unique restant dans la tumeur ont révélé une perte préférentielle d'allèles maternels pour le bras court du chromosome 11 dans 26 néphroblastomes sur 27. La probabilité que cette observation inattendue soit le fait du hasard a été estimée à moins de $0,1 \%$. Des observations identiques, une perte préférentielle d'al-

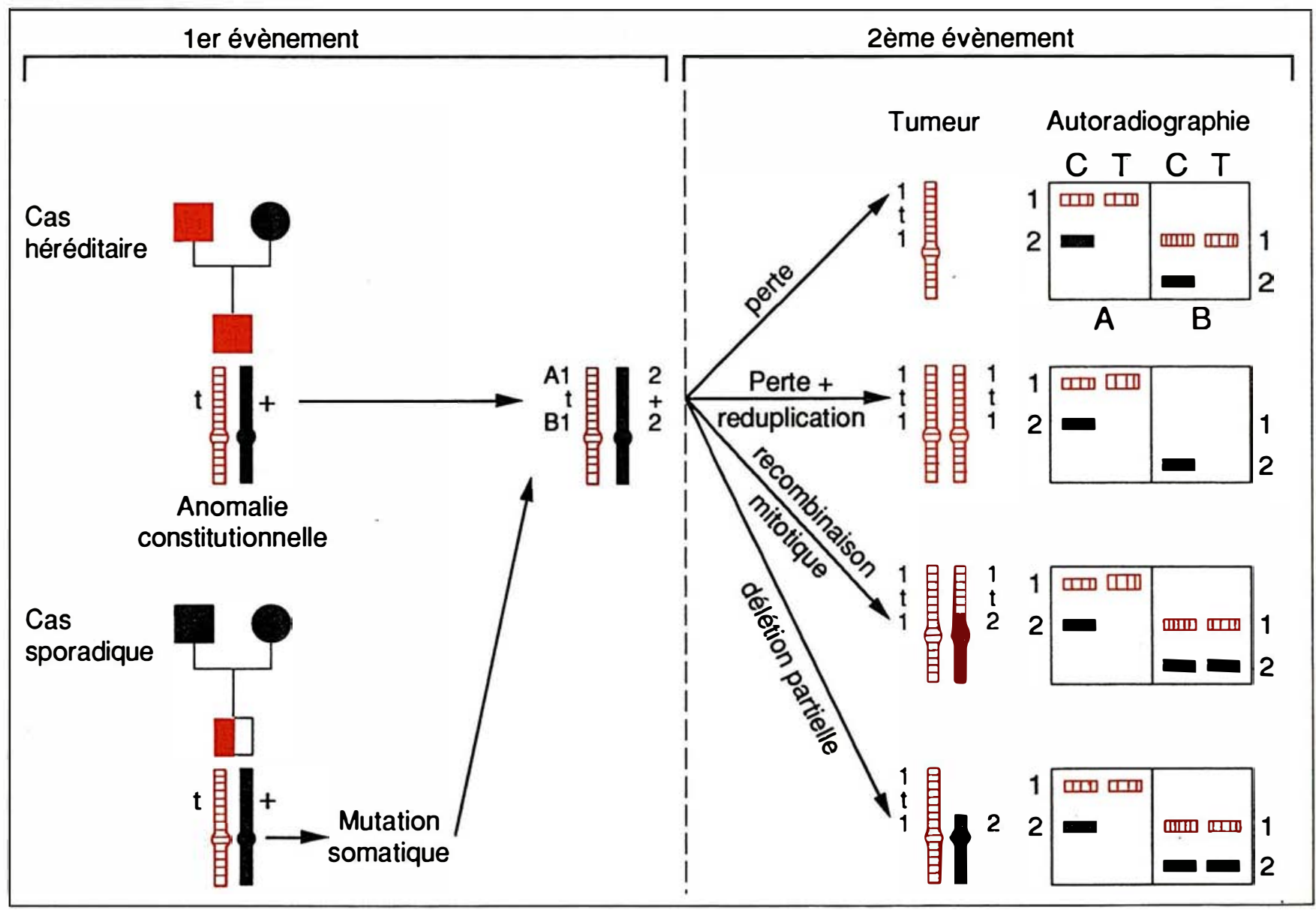

Figure 1. "Pertes d'allèles" ou «perte de l'hétérozygotie » dues à des remaniements chromosomiques somatiques. La comparaison des âges d'apparition de tumeurs de la rétine à caractère héréditaire et de tumeurs sporadiques a permis à Knudson de proposer la « théorie des deux événements». Dans les tumeurs héréditaires, une première mutation d'origine germinale est présente dans toutes les cellules de l'individu. II suffit qu'une deuxième mutation, somatique cette fois, se produise pour que la tumeur apparaisse. Dans les tumeurs sporadiques, il faudra que la deuxième mutation somatique se produise dans une cellule dans laquelle une première mutation se sera déjà produite. Représentation schématique des différents mécanismes impliqués dans la perte de l'hétérozygotie, montrant la comparaison des génotypes constitutionnels et tumoraux par la technique de Southern, pour les polymorphismes étudiés. $t=$ allèle altéré, $+=$ allèle sauvage, $A$ et $B$ $=$ séquences $A D N$ détectant des polymorphismes (RFLP), choisies le telle sorte que l'individu soit hétérozygote. 1 et $2=$ allèles des différents marqueurs. $C=A D N$ constitutionnel provenalit des lymphocytes du sujet, $T=A D N$ tumoral. En noir: origine maternelle. En traits hachurés : origine paternelle. En rouge: cellules ou chromosomes portant un allèle muté : dans les formes sporadiques, le propositus est une mosaïque, certaines des cellules portant la mutation, d'autres pas. Dans l'exemple choisi, l'allèle muté est d'origine paternelle, dans les formes sporadiques et familiales. En fait, dans les formes familiales, il pourrait aussi bien être d'origine maternelle. 
lèles maternels, ont été rapportées pour d'autres tumeurs, comme le rhabdomyosarcome et l'ostéosarcome (W. Cavenee, communication personnelle [13]).

\section{Marquage différentiel et tumorigenèse}

Le modèle de Wilkins. A la suite de ces observations, Wilkins proposa pour le néphroblastome un modèle faisant intervenir l'idée d'une empreinte parentale différentielle [14]. Selon ce modèle, le gène situé en llpl3 (WT1) se comporterait comme un anti-oncogène qui aurait pour fonction d'inhiber un gène transformant également situé sur le bras court du chromosome 11 . Ce modèle, qui ne disposait pas alors de toutes les données, n'est probablement pas correct, mais il n'en demeure pas moins que l'idée de l'empreinte parentale était très séduisante.

Une empreinte différentielle est imposée aux génomes paternel et maternel. Le concept du marquage et de l'expression différentiels des génomes paternel et maternel repose essentiellement sur des études portant sur le développement de l'embryon de souris mais également sur le mode d'expression de transgènes qui, dans certains cas, semble dépendre exclusivement du parent qui l'a transmis [15]. De nombreuses observations soulignent le caractère indispensable de la présence du génome maternel pour le développement de l'embryon, alors que le génome paternel est, lui, plus important pour le développement des annexes embryonnaires. Dans l'espèce humaine, l'élimination du génome maternel de l'œuf fécondé donne naissance à une mole hydatiforme qui peut à son tour se transformer en choriocarcinome. A cette complémentarité entre les génomes maternel et paternel, il faut également ajouter des interactions entre les génomes parentaux, particulièrement au début du développement. Si les processus de méthylation semblent impliqués dans le phénomène d'empreinte, ils ne sont sûrement pas les seuls en cause. On ne connaît ni le nombre, ni la nature, ni la séquence précise des événements conduisant à ce marquage, pas plus que l'existence éventuelle de processus de « gommage » de ces empreintes. Ce marquage différentiel ne toucherait qu'environ 6 à $12 \%$ du génome et bien qu'il semble, d'après des observations chez la souris, que des pans entiers du génome ne soient pas soumis à cette empreinte, il est, en revanche, impossible de dire si l'empreinte concerne un seul gène ou s'étend à plusieurs gènes contigus, voire à une région chromosomique entière. Il ne semble pas non plus que l'empreinte touche la totalité des cellules. Une revue sur le sujet a été récemment publiée dans médecine /sciences ( $n^{\circ} 1$, vol. 5, p. 8) [16].

\section{Néphroblastome : combien de gènes}

Longtemps considéré comme analogue au modèle du rétinoblastome, le modèle de la prédisposition à la tumeur de Wilms montre de plus en plus de divergences qui s'expliquent, en partie, par le fait que plusieurs gènes sont impliqués.

Au moins trois gènes de prédisposition. - Le néphroblastome est une tumeur rare de l'enfant avec une fréquence de $1 / 10000$. Environ $1 \%$ des sujets sont porteurs d'une délétion constitutionnelle de la région $11 \mathrm{pl} 3$ et présentent non seulement une prédisposition à développer un néphroblastome ou tumeur de Wilms (WT), mais également une aniridie (A), des anomalies génito-urinaires $(G)$, et une arriération mentale $(R)$, qui constituent le syndrome WAGR [17]. La taille de la délétion en 11 pl3 peut être variable et ainsi se traduire par un syndrome partiel. La pénétrance n'est pas complète: environ deux tiers des sujets présentant cette délétion développent un néphroblastome.

- Un autre syndrome malformatif, caractérisé par un gigantisme, un omphalocèle, une macroglossie, le syndrome de Beckwith-Wiedemann (BWS), est lui aussi associé à une prédisposition au néphroblastome [18]. Cette tumeur, quoique la plus fréquente, n'est pas la seule observée chez les sujets ayant un BWS. On observe également des corticosurrénalomes, des rhabdomyosarcomes et des hépatoblastomes [19].
13. Toguchida J, Ishizaki K, Sasaki MS et al Preferential mutation of paternally derived RB gene as the initial event in sporadic osteosarcoma. Nature 1989 ; 338 : 156-8. 
Tableau I

SITES DES MUTATIONS GERMINALES (C) ET SOMATIQUES (T) OBSERVÉES DANS DIFFÉRENTS CAS DE TUMEURS DE WILMS A CARACTĖRE HÉRÉDITAIRE (FAMILIAL, BILATÉRAL, WAGR ET BWS) OU BIEN A CARACTËRE SPORADIQUE

\begin{tabular}{|c|c|c|c|c|c|c|c|c|c|c|c|}
\hline \multicolumn{2}{|c|}{ Locus } & \multicolumn{10}{|c|}{ WT } \\
\hline & & \multicolumn{2}{|c|}{ Familiaux } & \multicolumn{2}{|c|}{ Bilatéraux } & \multicolumn{2}{|c|}{ WAGR } & \multicolumn{2}{|c|}{ BWS } & \multicolumn{2}{|c|}{ Sporadiques } \\
\hline & & C & $\mathrm{T}$ & C & $\mathrm{T}$ & C & $\mathrm{T}$ & C & $\mathrm{T}$ & C & $T$ \\
\hline WT2 & $11 \mathrm{p} 15$ & & oui & & oui & & oui & oui & oui & & oui \\
\hline WT1 & $11 p 13$ & & & & $?$ & oui & & & & & oui \\
\hline WT3 & autre & oui & & & & & & & & & \\
\hline
\end{tabular}

Bien que la plupart des cas soient sporadiques ou familiaux sans anomalie chromosomique, l'existence d'une douzaine d'observations avec duplication constitutionnelle de la région $1 \mathrm{lpl} 5.5$ laisse supposer qu'un gène porté par cette région est responsable des différentes formes. La région $1 \mathrm{lpl} 5.5$ est effectivement impliquée dans les formes familiales de BWS, puisque des études familiales réalisées à l'aide de marqueurs de cette région ont montré une liaison génétique [20, 21].

Parmi ces douze cas de BWS avec duplllpl5 décrits dans la littérature, un seul sujet a développé une tumeur, un corticosurrénalome. $\mathrm{Ce}$ type de tumeur étant fréquemment associée au BWS, on peut supposer que la prédisposition au corticosurrénalome est directement liée à l'altération d'une séquence située en $1 \mathrm{lpl} 5.5$ [22].

- Les formes familiales de WT ne représentent qu'environ $2 \%$ des cas. Deux groupes ont exclu la possibilité d'une liaison entre le locus de la prédisposition au néphroblastome dans ces familles et la région $11 \mathrm{pl} 3$ ainsi que la région $11 \mathrm{pl} 15.5[9,23]$. La localisation chromosomique de ce troisième locus, impliqué dans la susceptibilité à la WT, n'est pas encore connue.

- Trois régions distinctes portent donc des gènes impliqués dans la prédisposition au néphroblastome: le gène $W T 1$ situé en $1 \mathrm{lpl} 3$ associé au syndrome WAGR, le gène WT2 $\mathrm{m} / \mathrm{s} n^{\circ} 7$ vol. 5 , seplembre 89 localisé en 11 pl5.5 impliqué dans le BWS et un autre locus, WT3, associé aux formes familiales (Tableau I).

Pertes d'allèles: deux régions distinctes. C'est grâce à l'utilisation systématique d'un plus grand nombre de marqueurs permettant de mieux distinguer les différentes régions du bras court du chromosome ll, qu'il a été possible de démontrer que, pour un grand nombre de tumeurs, incluant des cas de WAGR, un cas familial et des cas sporadiques, les pertes d'allèles pouvaient être limitées à la région $1 \mathrm{lpl}$ 15.5. En ce qui concerne la région $11 \mathrm{pl} 3$, des pertes d'allèles impliquant à la fois cette région mais également la région 11 p15 ont été observées. En revanche, il n'est fait mention dans la littérature que d'un seul cas de néphroblastome sporadique présentant une perte d'allèle, en fait une délétion homozygote, limitée à la région $11 \mathrm{pl} 3$ [24]. Il existe donc deux régions, 11 pl 3 et 11 pl5.5, clairement distinctes sur le bras court du chromosome 11, impliquées dans les mécanismes d'initiation et/ou de progression tumorales correspondant très vraisemblablement aux loci WT1 et WT2 (Tableau I).

Le site pour lequel est observé une mutation somatique (perte d'allèles) n'est donc pas nécessairement le même que le site de la mutation germinale: l'observation de pertes d'allèles impliquant la région du gène WT2, dans des cas de WAGR

et une forme familiale de WT, est à rapprocher de ce qui a été rapporté pour d'autres tumeurs. Pour les cancers médullaires de la thyroïde, alors que le gène de susceptibilité $(M E N 2 A)$ se trouve sur le chromosome 10 [25], il n'a jamais été observé de perte d'allèles pour cette région dans les tumeurs. En revanche, des pertes d'allèles pour le chromosome l ont été observées [26]. De la même façon, le gène de la polypose colique familiale $(F A P)$ se trouve sur le chromosome 5 , mais les pertes d'allèles observées dans des carcinomes colorectaux sporadiques et familiaux touchent beaucoup plus fréquemment les chromosomes 17 et 18 que le 5 [27].

\section{Anti-oncogène ou gène transformant?}

L'empreinte parentale différentielle des génomes d'origine maternelle ou paternelle peut avoir pour conséquence: (1) soit l'hyperméthylation des cytosines de l'un des génomes est à l'origine d'une hypermutabilité. Dans le cas des néphroblastomes, elle concernerait les allèles paternels. La première mutation, dans les cas sporadiques, surviendrait donc préférentiellement sur les allèles paternels. La perte consécutive de l'allèle maternel étant à l'origine de la tumeur. Il n'est pas impossible que dans les cas héréditaires, la première mutation se produise également sur un allèle paternel, pour être ensuite transmise indifféremment par une femme ou un homme. L'étude, dans des familles, de l'origine parentale des gamètes porteurs d'une néomutation devrait permettre de vérifier cette dernière hypothèse; (2) soit l'empreinte parentale se traduit par une modulation différente de l'expression des deux allèles, dans le temps et/ou dans l'espace. La perte du seul allèle «actif »'d'un gène de type anti-oncogène suffirait et serait à l'origine du processus tumorigène, ou bien la perte de l'allèle « inaçtif » suivie d'une reduplication de l'allèle actif d'un oncogène se traduirait par une transformation due à un effet de dose. Dans cette hypothèse, pour les cas familiaux, les phénotypes des sujets devraient être différents selon qu'un sujet aura reçu le gène muté de son père ou de sa mère. 
Il existe cependant d'autres mécanismes possibles qui feraient intervenir un plus grand nombre d'événements. En effet, contrairement à ce que l'on pouvait attendre, d'après la théorie des deux événements au même locus, les différents cas de figure observés montrent que le modèle du néphroblastome est certainement plus complexe que le modèle du rétinoblastome.

Délétion homozygote de WT1/11pl3 dans un cas sporadique. Un grand nombre de tumeurs ont été étudiées avec des marqueurs de la région $1 \mathrm{lpl} 3$ et seul un cas de WT sporadique a montré une délétion homozygote pour un marqueur, $\mathrm{Sl}$, situé à proximité du locus WTI [24]. La délétion des deux copies du gène $W T 1$ en 1 lpl3 semble donc étrangement rare par rapport aux pertes d'allèles en 1 lpl5.5 que l'on trouve dans un grand nombre de tumeurs. Cela peut être mis au compte de l'existence, dans cette région, d'un grand nombre de gènes dont l'absence serait létale pour la cellule ou de mutations ponctuelles qui n'auraient pu être détectées [28]. Cette observation, bien qu'unique, indique que le gène $W T 1$ en llpl3 se comporte bien comme un anti-oncogène analogue au gène du rétinoblastome [24] (figure 2A). Il ne peut être question d'envisager l'hypothèse d'une empreinte parentale avec ce seul cas.

WAGR et perte d'allèles en 11 pl5.5. Les tumeurs de trois sujets avec délétion germinale en 11 pl3 ont été étudiées avec des marqueurs couvrant la totalité du chromosome 11. Dans les trois cas, une perte d'allèles limitée à la partie distale de la région 11 p 15.5 , plus précisément la région $11 \mathrm{p} 15.5$ portant $W T 2$, a été observée [29]. Il n'est toutefois pas possible d'exclure une mutation ponctuelle, altérant l'unique copie du gène $W T I$ en l lp13. Cela ne sera effectivement possible que lorsque le gène $W T I$ sera cloné (figure $2 B$ ). L'étude des parents, qui a pu être réalisée dans un des cas (figure 3, cas AZA), a permis de montrer que l'allèle perdu dans la tumeur est encore d'origine maternelle. Afin de déterminer si l'événement mitotique était une délétion avec perte d'une copie ou bien

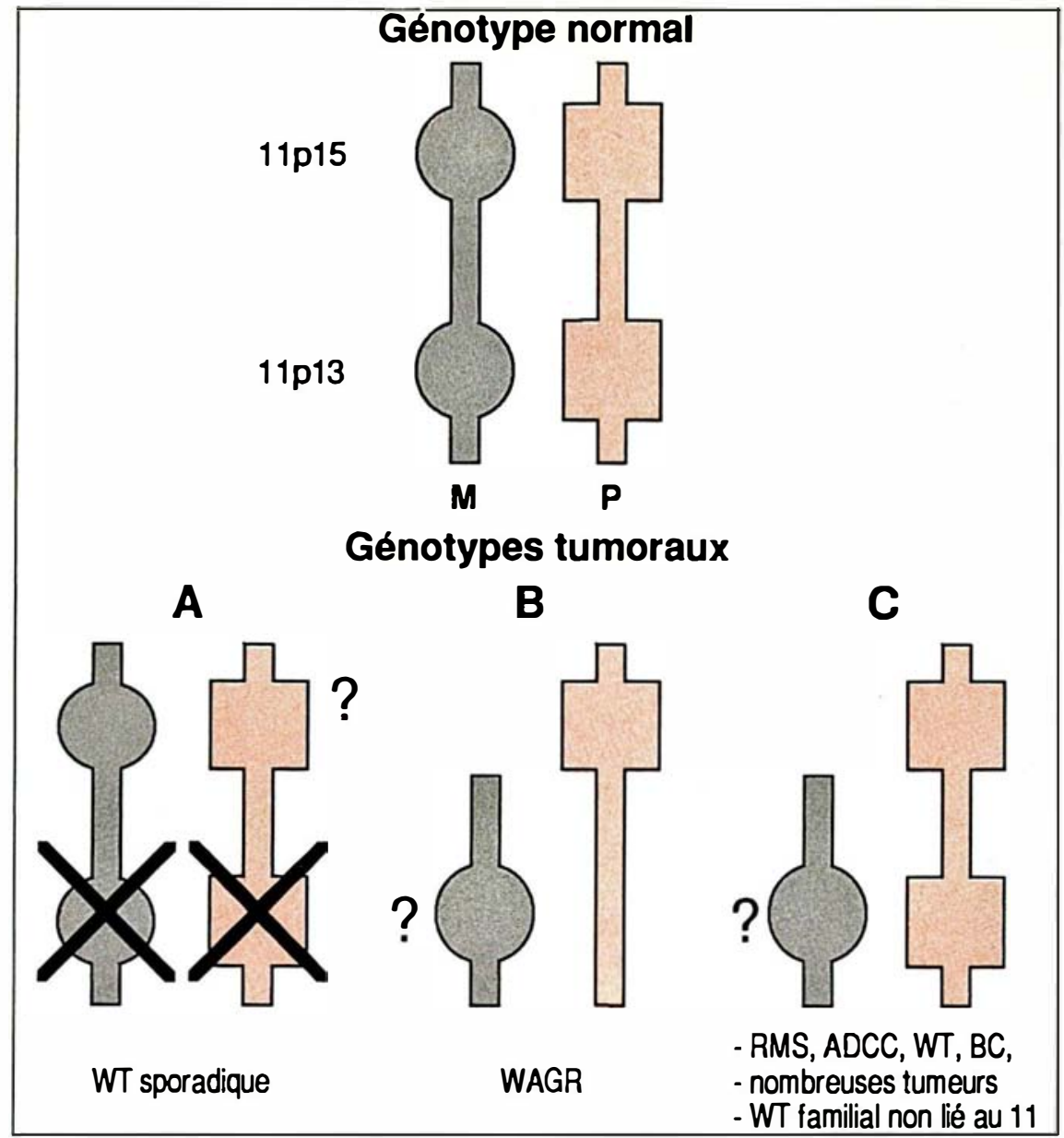

Figure 2. Différentes situations observées dans les tumeurs et faisant intervenir les régions 11 p13 et 11 p15.5. A. Un cas de délétion homozygote en 11p 13 dans une forme sporadique, sans perte d'allèle pour WT2 en 11p15.5, mais n'excluant pas une mutation ponctuelle non détectable en WT2. B. Trois cas de WAGR avec délétion constitutionnelle en 11p13 et perte d'allèles (délétion ou recombinaison mitotique) en 11p15.5. Un seul cas, informatif, a montré une perte de l'allèle maternel en 11p15 dans la tumeur (cas AZA, voir figure 3). C. Nombreux cas de différentes tumeurs avec perte préférentielle de l'allèle maternel en 11p15.5. Là encore, il n'est pas possible d'exclure une mutation ponctuelle pour WT1 en 11p13. $M=$ maternel ; $P=$ paternel.

deux copies identiques, le nombre de copies des allèles restants dans la tumeur en 1 lpl5.5 a été dosé. Sur les trois cas de WAGR étudiés, il s'agissait de délétion dans deux cas (cas BAN et ZER) et de recombinaison mitotique dans un cas (cas AZA) (figure 3) [29].

Ces résultats montrent que le site (en $1 \mathrm{lpl}$ 5.5) pour lequel une perte d'allèles est observée peut être différent du site de la mutation germinale, défini sans ambiguïté ici, puisqu'il s'agit de trois sujets avec delllpl3 constitutionnelle. Dans la première hypothèse, celle d'un anti-oncogène régulateur soumis à empreinte parentale, il faut supposer que l'allèle maternel WT2 perdu est l'allèle «actif» alors que l'allèle paternel WT2, restant, est inactivé. Cette inactivation pouvant être due à une empreinte parentale ou à une mutation elle-même secondaire à une empreinte parentale. Dans la seconde hypothèse, celle d'un gène transformant, il faudrait pouvoir invoquer un effet de dose; or l'existence de 


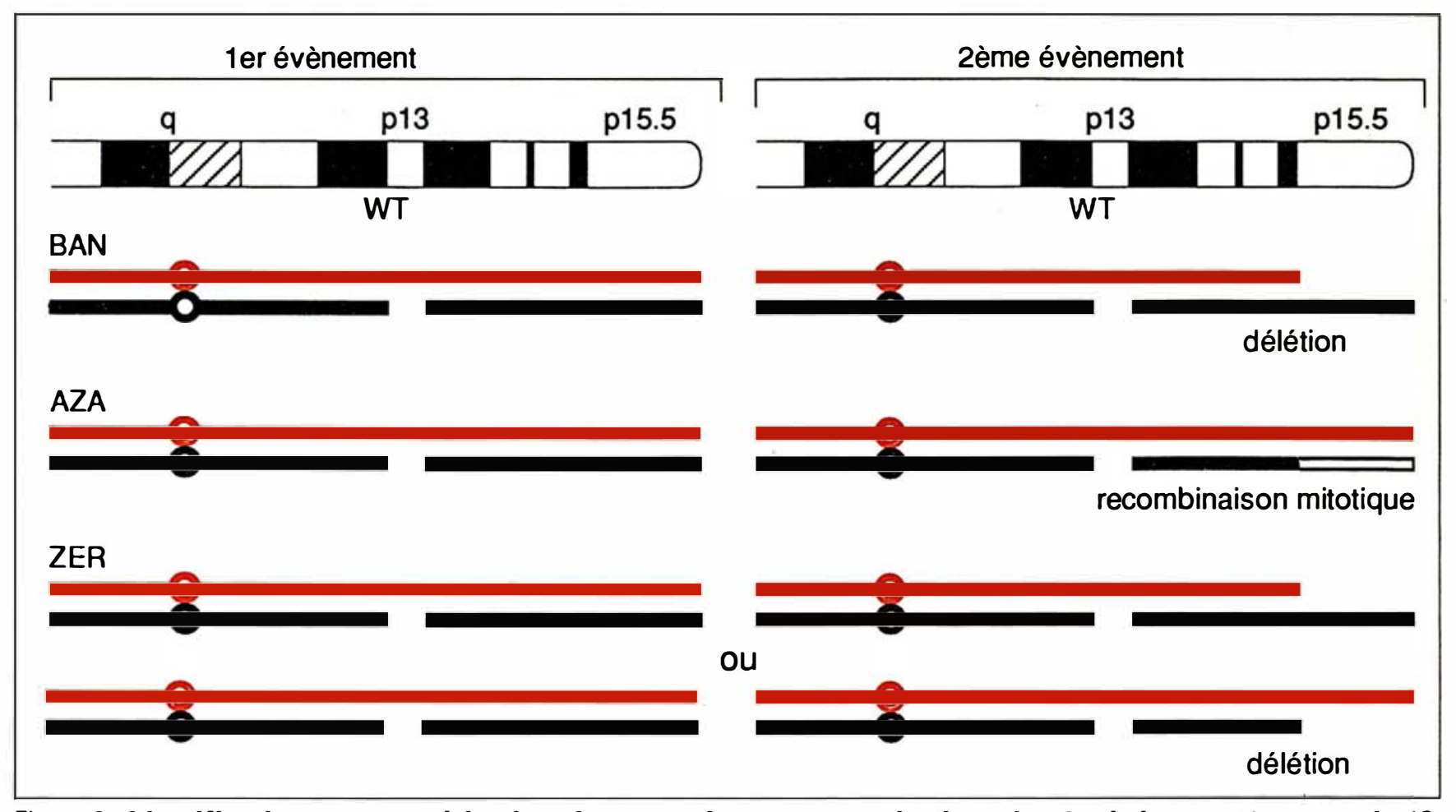

Figure 3. Identification et caractérisation des remaniements constitutionnels (10r événement) et acquis (20 événement) dans trois cas de WAGR avec délétion constitutionnelle en 11 p13 et perte d'allèles en $11 p 15.5$ dans la tumeur. Dans le premier cas (BAN), la réalisation d'hybrides somatiques a permis de montrer que la perte d'allèles, due à une délétion en 11p15.5, touchait le chromosome normal non porteur de la délétion constitutionnelle en 11p13 [21]. Dans le deuxième cas (AZA), l'étude familiale a montré que la délétion constitutionnelle en $11 p 13$ ainsi que la perte d'allèles en 11p15.5, due ici à une recombinaison mitotique, touchait le même chromosome, le chromosome d'origine maternel. Dans le troisième cas (ZER), en l'absence d'hybrides somatiques et d'étude familiale, il n'a pas été possible d'identifier l'origine du chromosome ayant subi les remaniements.

délétions somatiques (cas BAN et ZER) va à l'encontre de cette proposition. La seule possibilité serait donc celle d'une mutation sur l'allèle paternel, lui conférant un pouvoir transformant.

Formes familiales de WT et perte d'allèles en 1lp15.5. Là encore, c'est le locus WT3 responsable des formes familiales qui devrait être impliqué dans les pertes d'allèles. Pourtant, l'étude d'une tumeur dans une de ces familles a révélé, à nouveau, une perte de l'hétérozygotie limitée à la région $11 \mathrm{pl} 5.5$ [9]. Le site observé pour la mutation somatique, WT2 en 11 pl5.5, est donc comme pour les trois cas de WAGR, différent du site de la mutation germinale (WT3) responsable de la prédisposition. Des pertes préférentielles d'allèles maternels pour la totalité du bras court du $\mathrm{m} / \mathrm{s} n^{\circ} 7$ vol. 5 , septembre 89 chromosome 11 ont également été retrouvées dans des cas familiaux [11] (figure 2C).

Ces observations vont dans le même sens que les observations précédentes en ce qui concerne le locus WT2, qui de plus est soumis à une empreinte parentale. Pour le locus WT3, il serait prématuré d'émettre une hypothèse concernant une éventuelle empreinte parentale.

Syndrome BWS et perte d'allèles en 1 lp15.5. L'étude de tumeurs survenues chez des sujets atteints de BWS, sans anomalie constitutionnelle en $1 \mathrm{lpl}$ 15.5, a montré une perte d'allèles pour la région $11 \mathrm{pl} 5.5$ dans des néphroblastomes [10] et un hépatoblastome [30]. La même anomalie a été trouvée dans un adénome de la surrénale [31]. Il n'existe pas de données concernant l'origine parentale (figure $2 C$ ).

Ces observations semblent indiquer que le gène $W T 2$ peut donc, lui aussi, se comporter comme un antioncogène.

Autres tumeurs avec perte d'allèles en 1lpl5.5. Bien qu'il n'existe pas d'indication formelle concernant le site de la première mutation dans différentes tumeurs sporadiques ou familiales comme les rhabdomyosarcomes, ou les corticosurrénalomes, mais du fait que ces tumeurs apparaissent souvent en association avec le syndrome de BWS, on pouvait s'attendre à des pertes d'allèles pour la région $11 \mathrm{pl}$ 15.5. Des délétions et des recombinaisons mitotiques limitées à la région $11 \mathrm{pl} 15.5$ ont été observées dans ces tumeurs ainsi que dans de nombreuses autres tumeurs comme le cancer du sein [32] et le 


\section{RÉFÉRENCES}

14. Wilkins RJ, Genomic imprinting and carcinogenesis. Lancet 1988 ; 1 : 329-31.

15. Swain JL, Stewart TA, Leder P. Parental legacy determines methylation and expression of an autosomal transgene : a molecular mechanism for parental imprinting. Cell $1987 ; 50: 719-27$

16. Babinet C, Barra J, Renard JP. Le marquage et l'expression différentiels des génomes paternel et maternel. médecinel sciences $1989 ; 5: 8-15$.

17. Francke U, Holmes LB, Atkins L, et al. Aniridia-Wilms tumor association : evidence for specific deletion of llpl3. Cytogenet Cell Genet 1979 ; 24 : 185-92

18. Beckwith JB. Extreme cytomegaly of the adrenal fetal cortex, omphalocele, hyperplasia of kidney and pancreas, and Leydig-cell hyperplasia: another syndrome? Los Angeles: Western Society for Pediatric Research, 1983.

19. Wiedemann HR. Tumors and hemihypertrophy associated with Wiedemann-Beckwith syndrome. Eur J Pediatr 1983 ; 141 : 129.

20. Ping AJ, Reeve AE, Law DJ, et al. Gene(ic linkage of Beckwith-Wiedemann syndrome to Ilpl5. Am J Hum Genet 1989 ; 44 : 720-3.

21. Koufos A, Grundy P, Morgan K, et al. Familial Wiedemann-Beckwith syndrome and a second Wilms tumor locus both map to Ilpl5.5. Am J Hum Genet 1989; $44: 711$ 9.

22. Henry I, Jeanpierre $\mathrm{M}$, Couillin $\mathrm{P}$, et al. Molecular definition of the 11 pl5.5 region involved in Beckwith-Wiedemann syndrome and in predisposition to adrenocortical carcinoma. Hum Genet 1989; 81 : 273-7.

23. Huff V, Compton DA, Chao LY, et al. Lack of linkage of familial Wilms tumor to chromosomal band 1lp 13. Nature 1988; 336 : 377-8.

24. Lewis WH, Yeger $\mathrm{H}$, Bonetta $L$, et al. Homozygous deletion of a DNA marker from chromosome $11 \mathrm{pl} 3$ in sporadic Wilms tumor. Genomics 1988 ; 3 : 87-90.

25. Mathew CG, Chin KS, Easton DF, et al. A linked genetic marker for multiple endocrine neoplasia type $2 \mathrm{~A}$ on chromosome 10 . cancer de la vessie [33] (figure 2C). Une perte préférentielle d'allèles maternels souvent limitée à 11 pl5.5 a été observée dans cinq cas sur cinq de rabdomyosarcomes. Pourtant, dans certains cas, les types de remaniements observés et les marqueurs utilisés ne permettaient pas toujours de discerner la région, $11 \mathrm{pl} 3$ ou 11 pl5.5, réellement impliquée dans ce phénomène.

Il est donc clair, à travers ces données, que l'hypothèse de Knudson ne permet plus d'expliquer toutes les observations.

\section{Le modèle souris}

Il existe, comme nous allons le voir, un certain nombre d'arguments fondés sur des observations chez l'homme et chez la souris qui renforcent l'idée d'une empreinte différentielle selon l'origine parentale et selon la région du bras court du chromosome 11 (1lpl3 ou llpl5.5).

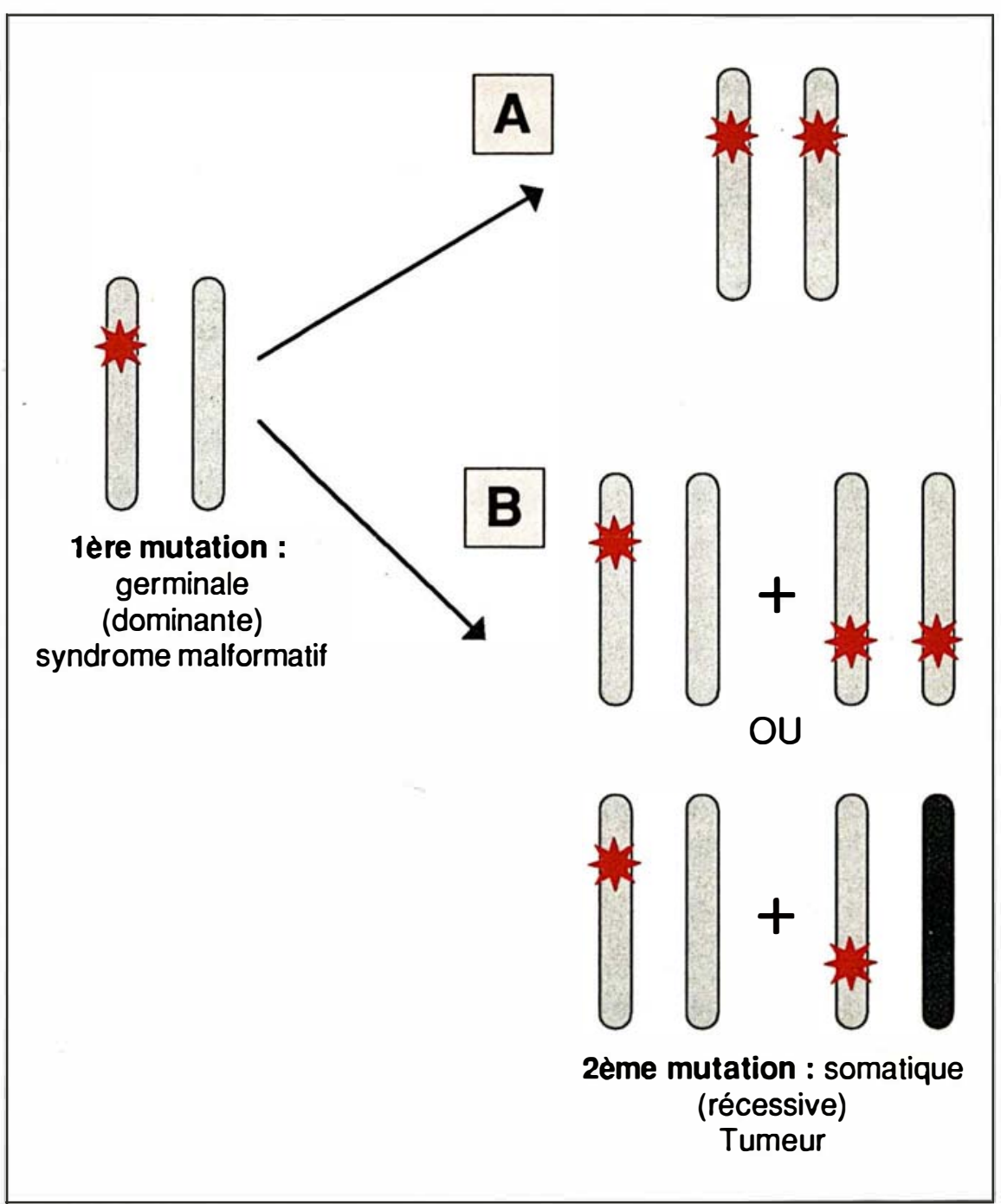

Figure 4. Nombre, nature et localisation des événements successifs conduisant à l'apparition d'une tumeur. Dans les cas héréditaires associant ou non un syndrome malformatif, la première mutation est soit muette soit responsable du syndrome malformatif. La tumeur se développe lorsqu'une deuxième mutation touche le deuxième allèle du même gène (A). La deuxième mutation peut, dans certains cas (B), se produire à un locus différent soumis ou non à une empreinte parentale (chromosome noir). Des exemples de ces deux types de situation figurent dans le Tableau II. 
Les progrès considérables réalisés au cours de ces dernières années dans l'établissement de la carte chromosomique de l'homme et de la souris ont permis de reconnaître l'existence de nombreux groupes de liaison conservés chez les deux espèces, mais souvent dispersés sur des chromosomes différents. Il faut cependant souligner que le degré de précision des localisations géniques, et donc des régions soumises à l'empreinte parentale différentielle, n'est pas la même chez l'homme et la souris. Cela doit donc inciter à une certaine prudence dans l'extrapolation des résultats d'un génome à l'autre. Certaines régions du bras long et du bras court du chromosome ll humain (llpl5.5) se trouvent sur le chromosome 7 de la souris, alors que la région médiane du bras court, la région $1 \mathrm{lpl} 3$, se trouve sur le chromosome 2 de la souris [34]. Il est intéressant de noter que des pertes d'allèles pour la région du chromosome 7 de la souris correspondant très précisément à la région humaine 11 pl5.5 ont également été retrouvées dans des carcinomes de souris [35]. Si l'on examine maintenant la carte de la souris montrant les régions chromosomiques intéressées par un phénomène d'empreinte parentale [36], il est frappant de constater que la région du chromosome 7 murin homologue de la région $1 \mathrm{lpl} 5.5$ humaine est soumise à un marquage différentiel. Il y a défaut de complémentation en cas de duplication paternelle/absence maternelle aussi bien qu'en cas de duplication maternelle/absence paternelle. A l'inverse, la région du chromosome 2 murin portant la région homologue de la région $1 \mathrm{lpl} 3$ humaine montre une complémentation normale, excluant tout phénomène d'empreinte. Il faut bien entendu, là encore, rester prudent dans l'extrapolation.

\section{Prédisposition / pertes d'allèles / empreinte parentale différentielle}

L'altération de l'un des trois gènes $W T 1, W T 2$, et $W T 3$, peut donc être responsable d'une prédisposition à la WT (ou à d'autres tumeurs dans le cas de WT2). Cependant, en dépit de l'hypothèse proposée par $\mathrm{m} / \mathrm{s} n^{\circ} 7$ vol. 5 , septembre 89
Comings [6], selon laquelle les deux événements doivent se produire sur le même locus, ces exemples suggèrent que le premier événement a un effet dominant responsable du syndrome malformatif lui-même, WAGR pour WT1 et BWS pour WT2. Quant au deuxième événement, favorisé par un état de différenciation et/ou de prolifération anormales, il peut se produire sur le même locus ou sur un voire plusieurs loci différents (figure 4). Ainsi, la délétion du gène $W T 1$ en 11 pl3 pourrait être uniquement responsable d'anomalies génito-urinaires, la perte du gène WT2 soumis à une empreinte parentale étant, elle, indispensable au développement de la tumeur. Cela semble également être le cas dans les cancers médullaires de la thyroïde et le syndrome $M E N 2 A^{*}$, le carcinome colorectal et la polypose colique familiale ou bien encore les cancers du sein (Tableau II).

Les données actuelles ne permettent pas de trancher pour le gène $W T 2$ entre les deux hypothèses «antioncogène » ou « oncogène ».

- Il existe pourtant de solides arguments en faveur de la nature antioncogène de ce gène: le gène $W T 2$ peut effectivement être un gène suppresseur, indépendant de $W T 1$, et dont la simple perte de l'allèle maternel actif suffirait à enclencher le processus tumorigène. Ceci corroborerait d'ailleurs la fréquence étonnante des pertes d'allèles pour cette région, dans des tumeurs diverses. Dans le cancer colorectal, les pertes d'allèles pour les régions des chromosomes 5 ,

* MEN2A : multiple endocrine neoplasia typeIIA associant cancer médullaire de la thyroïde et phéochromocytome.

Tableau ॥

LOCALISATION DES GĖNES DE SUSCEPTIBILITÉ

AU CANCER, ASSOCIÉS OU NON A UN SYNDROME MALFORMATIF, ET PERTES D'ALLELLES DANS LES TUMEURS

\begin{tabular}{|c|c|c|}
\hline & $\begin{array}{l}\text { Gène de } \\
\text { prédisposition }\end{array}$ & $\begin{array}{l}\text { Pertes } \\
\text { d'allèles }\end{array}$ \\
\hline 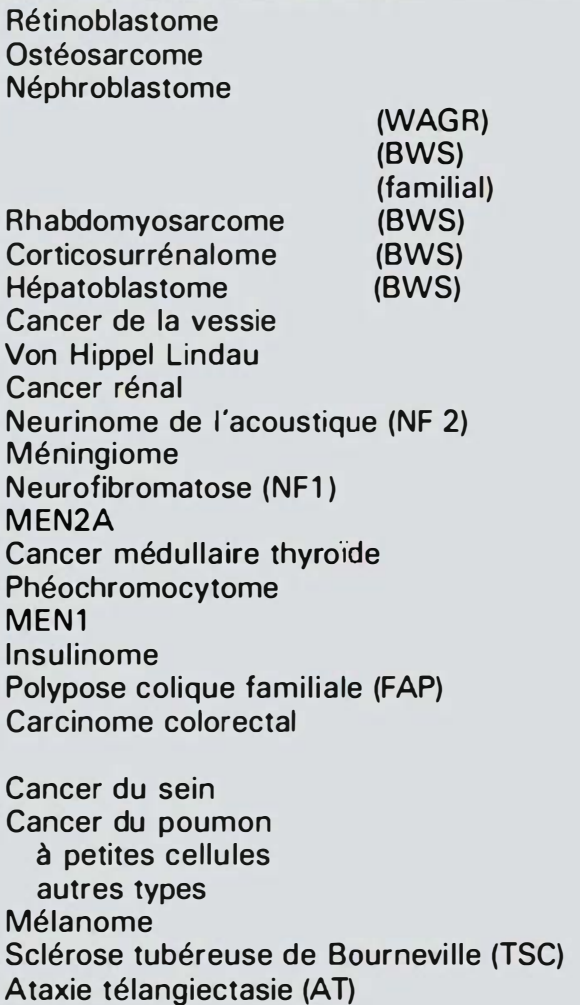 & $\begin{array}{l}13 q 14 \\
13 q 14 \\
11 p 13 \\
11 p 15 \\
\text { autre? } \\
11 p 15 \\
11 p 15 \\
11 p 15 \\
? \\
3 p \\
22 q \\
17 q 11 \\
10 q \\
11 q \\
5 q 21 \\
?\end{array}$ & $\begin{array}{l}13 q 14 \\
13 q 14 \\
11 p 13,11 p 15 \\
11 p 15 \\
11 p 15 \\
11 p 15 \\
11 p 15 \\
11 p 15 \\
11 p 15 \\
11 p 15 \\
3 p \\
22 q \\
1 p \\
1 p, 22 \\
11 q \\
5 q, 17 p, 18 p \text {, } \\
22 \\
13 q, 11 p \\
3 p, 13 q, 17 p \\
3 p(13 q, 17 p) \\
\text { multiples }\end{array}$ \\
\hline
\end{tabular}




\section{RÉFÉRENCES}

26. Mathew CG, Smith BA, Thorpe K, et al. Deletion of genes on chromosome 1 in endocrine neoplasia. Nature 1987 ; 328 : 524-6.

27. Vogelstein B, Fearon ER, Stanley BA, et al. Genetic alterations during colorectal tumor development. $N$ Engl J Med 1988; 319: 525-32.

28. Bickmore WA, Porteous DJ, Christie S, et al. CpG islands surround a DNA segment located between translocation breakpoints associated with genitourinary displasia and aniridia. Genomics (soumis pour publication).

29. Henry I, Grandjouan S, Couillin P, et al. Tumor specific loss of 11 pl5.5 alleles in delllpl3 Wilms tumor and in familial adrenocortical carcinoma. Proc Natl Acad Sci USA 1989 (sous presse).

30. Little $\mathrm{MH}$, Thomson DB, Hayward NK et al. Loss of alleles on the short arm of chromosome 11 in a hepatoblastoma from a child with Beckwith-Wiedemann syndrome Hum Genet 1988; 186-9.

31. Hayward NK, Little MH, Mortimer RH, et al. Generation of homozygosity at the c Ha-ras-l locus on chromosome $11 p$ in an adrenal adenoma from an adult with Wiedemann-Beckwith syndrome. Cancer Genet Cytogenet $1988 ; 30: 127-32$.

32. Ali IU, Lidereau $\mathrm{R}$, Theillet $\mathrm{C}$, et al. Reduction to the homozygosity of genes on chromosome 11 in human breast neoplasia. Science $1987 ; 238$ : 185-8.

33. Fearon ER, Feinberg AP, Hamilton $\mathrm{SH}$ et al. Loss of genes on the short arm of chromosome 11 in bladder cancer. Nature $1985 ; 318$ : $377-80$.

34. Davisson MT, Roderick TH. Locus map of the mouse. Cytogenet cell Genet 1987; 1 (4) : 377-9.

35. Bremmer RA, Balmain A. Molecular genetic of tumour progression in mouse skin carcinogenesis. Genome 1988; 30 (suppl. 1) 197 (abstr.).

36. Beechey CV, Cattanach BM, Searle AC. Genetic imprinting map. Mouse News Lett $1988 ; 81$ : 48-9.

37. Sapienza C, Peterson AC, Rossant J, Balling $R$. Degree of methylation of transgens is dependent on gamete of origin. Nature 1987 ;
17 et 18 observées, se produisent de façon aléatoire et traduisent vraisemblablement la perte successive de différents « verrous », aboutissant à une absence totale de contrôle.

- Cependant, il n'est pas possible d'écarter formellement l'hypothèse d'un gène de type oncogène régulé par un autre gène régulateur agissant en trans: ce gène (ou ces gènes) situé(s) en 11 pl3 (WTI) ou ailleurs (WT3), qui pourrai(en)t être altéré(s) au cours du processus tumorigène, aurai(en)t pour fonction normale de réguler l'expression du gène $W T 2$ situé en $1 \mathrm{lpl}$ 5.5. Une coopération entre les produits de ces gènes n'est pas impossible non plus. Il pourrait également exister des interactions entre les allèles maternel et paternel dont l'équilibre serait rompu lors de la perte des allèles maternels dans la tumeur.

Il n'existe pas encore d'arguments permettant de penser que l'empreinte parentale a pour seul effet de diminuer, voire de supprimer, l'expression de l'allèle d'origine paternelle. L'hyperméthylation qui accompagne l'empreinte parentale peut être à l'origine d'une hypermutabilité responsable de mutations plus fréquentes sur les allèles d'origine paternelle; la tumeur n'apparaissant alors qu'après effaçage de l'empreinte et perte de l'allèle maternel «normal ». Il n'est pas impossible en effet, comme le suggère Sapienza [37], que cette empreinte ne soit pas universelle, ou bien qu'elle s'efface en partie au cours du développement. Bien que la méthylation ne soit probablement pas le seul mécanisme en cause, il devrait maintenant être possible d'explorer l'état de méthylation des allèles d'origine parentale différente, dans le rein normal, dans des îlots de néphroblastomatose considérés comme des lésions précancéreuses, et enfin dans la tumeur elle-même.

Alors que les recherches sur le cancer nous font découvrir de plus en plus la complexité des mécanismes de régulation cellulaire, il semblerait que la nature s'octroie, pour une fois, le droit d'être économe puisque, grâce à l'empreinte parentale, il ne serait plus nécessaire de perdre les deux allèles d'un anti-oncogène : la perte d'un seul allèle suffirait

\section{Summary}

Short arm of chromosome 11: genomic imprinting tumorigenesis and losses of alleles

The observation of a preferential loss of maternal alleles for region $1 \mathrm{lpl} 15.5$ suggests that the $1 \mathrm{lpl} 15.5$ gene which is involved not only in Wilms tumor (WT), but also in other tumors undergoes a differential parental imprinting. Imprinting could result either in hypermutability due to hypermethylation or in the absence of expression of one allele thus only requiring the loss of the unique active allele. There are at least three different loci implicated in hereditary predisposition to nephroblastoma (WT), one in $1 \mathrm{lpl} 3$ (WTl), one in $11 \mathrm{pl} 5$ (WT2) and a third one (WT3) as yet unmapped. Moreover, in some hereditary cases for which the site of the germinal mutation was known, the site for which a loss of alleles was found in the tumor was different from the site of the germinal mutation. This precludes identification of the location of the gene involved in hereditary predisposition by demonstrating loss of heterozygosity. The WT gene located in 11 pl3 has antioncogene features. Several schemes which may require interactions between repressor as well as activator genes can be proposed.

\section{TRÉS A PART}

Cl. Junien 